\section{IN THE NEWS}

Caffeine - a new cancer cure?

"Chocolate, cola and coffee could form the basis of new anticancer drugs, scientists believe" (BBC News).

Peter Shepherd and

colleagues say that the active ingredients in these, caffeine and theophylline, might be effective in fighting cancer because they target phosphatidylinositol 3-kinase (PI3K), a signalling molecule that regulates cell motility and survival. Many

pharmaceutical companies are in the process of developing agents that target PI3K as cancer therapeutics.

Researchers expressed the p110 $\delta$ subunit of PI3K in insect cells, and showed that its lipid-kinase activity could be inhibited by both caffeine and theophylline.

But before you rush off to buy more chocolate, be warned that the study involved "high concentrations of caffeine that would be unhealthy for human use. Caffeine has well-known side effects that make it inappropriate for drug use" (The Guardian).

The next step will therefore be to develop compounds that mimic the structure of caffeine without the negative effects.

Emma Greenwood

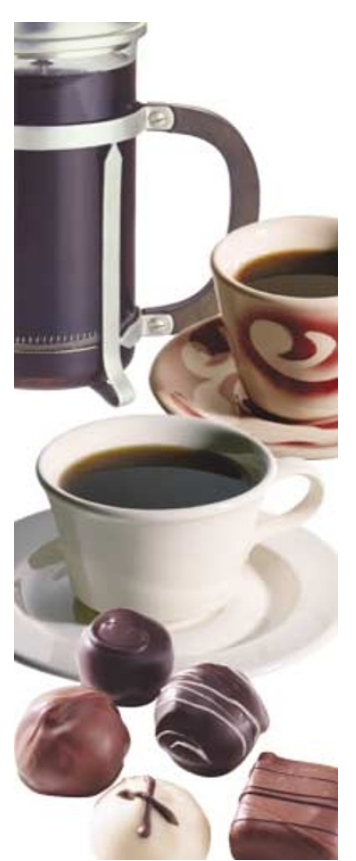

DNA REPAIR

\title{
Small cause, large effect
}

The Mre11 complex is a multisubunit nuclease that contains three components - Mre11, Rad50 and Nbs1 (known as Xrs2 in yeast). It acts as a sensor of double-stranded DNA breaks, but also has roles in checkpoint signalling and DNA replication. Research into the individual components has been hampered by the fact that null mutants are not viable, but John Petrini and colleagues now present their studies of a functionally impaired Rad50 mutant. And the results underline the importance of the Mre11 complex in the homeostasis of proliferative tissues.

Reporting in Genes and Development, Petrini and co-workers describe the generation of a group of hypomorphic Rad50 mutants, one of which - Rad50 $0^{K 22 M}$ - was used to derive so-called $R a d 50^{S / s}$-mutant mice. Rad50 $0^{S / S}$ mouse-embryo fibroblasts contained wild-type levels of Mre11, Nbs1 and Rad50 $0^{\mathrm{K} 22 \mathrm{M}}$, and

\section{DRUG METABOLISM}

\section{Not so innocent}

St. John's wort has been used as a medicinal plant for centuries and it has been widely assumed that, as a herbal preparation, it is harmless. However, in 2000, a seminal paper in The Lancet showed that the herb lowers plasma concentrations of indinavir, a protease inhibitor that is used to treat patients with HIV. Now, Mathijssen and colleagues, reporting in the 21 August issue of the Journal of the National Cancer Institute, show that it also interacts with a drug that is used to treat cancer, mitigating its effects in a similar way.

St. John's wort is thought to alleviate mild to moderate depression and is used widely among cancer patients. However, it is now recognized that the these components could assemble functional complexes. However, the Rad50 $0^{S / s}$ mice showed partial embryonic lethality, and those that survived were only $60 \%$ of the weight of their wild-type littermates. By $4-8$ weeks of age, the mice showed signs of anaemia, and most of them died by 4 months. Of those that survived up to 7 months, $20 \%$ died from a variety of tumours.

As the premature death was associated with severe anaemia, the authors first looked more closely at haematopoietic cells in the Rad50 $5 / 5$ mice. At 2 weeks, there was no difference between wild-type and Rad50 $0^{\mathrm{S} / \mathrm{S}}$ mice, but by 4 weeks of age, lymphocytes, macrophages, red blood cells and platelets were severely depleted in the mutant mice. Further experiments indicated that the progressive depletion was due to the failure of haematopoietic stem cells. More over, a similar depletion of cells was

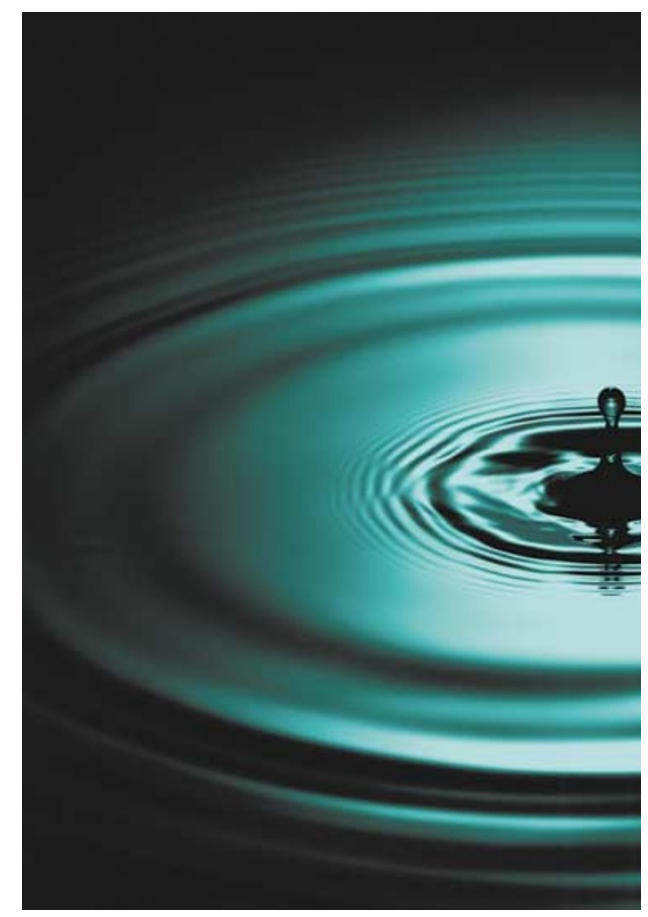

observed in the spermatogenic lineages of $R a d 50^{S / s}$ mice.

Given the age-dependent cellular depletion in the bone marrow and testes, and the fact that those mice that survived longest developed tumours, Petrini and co-workers wondered whether the Rad50 ${ }^{K 22 M}$ mutation might cause genotoxic stress. If this were the case, mutation in the gene that encodes p53 - which is involved in the response to genotoxic stress - herbal medicine induces drug detoxification pathways - the cytochrome P450 enzyme system and the P-glycoprotein drug transporter - and so interferes with the metabolism of classes of drugs that are substrates for these pathways.

Mathijssen et al. conducted a small randomized study of five cancer patients, treating them with irinotecan with or without St. John's wort. Irinotecan is, in part, eliminated by routes that are mediated by an isoform of $\mathrm{P} 450$, CYP3A4. The authors report that plasma levels of the active metabolite, $\mathrm{SN}-38$, were decreased by $42 \%$ after co-treatment with St. John's wort and that myelosuppression was also greatly reduced - about $60 \%$ with irinotecan alone but less than $8 \%$ with the combination. As irinotecan has a narrow therapeutic index, decreased plasma concentrations might be expected to lead to loss of antitumour activity.

The authors conclude that irinotecan and St. John's wort should not be given in combination. They hypothesize that these results are probably representative of other anticancer drugs that are at least partial substrates for CYP3A4, such as etoposide, tamoxifen and paclitaxel. The understanding of drug metabolism and mechanism of action of herbal preparations, and their interactions, are therefore highlighted as key areas of cancer research.

Ezzie Hutchinson

(2) References and links ORIGINAL RESEARCH PAPER Mathijssen, R. H. J. et al. Effects of St. John's wort on irinotecan metabolism. J. Natl Cancer Inst. 94 1247-1249 (2002) 


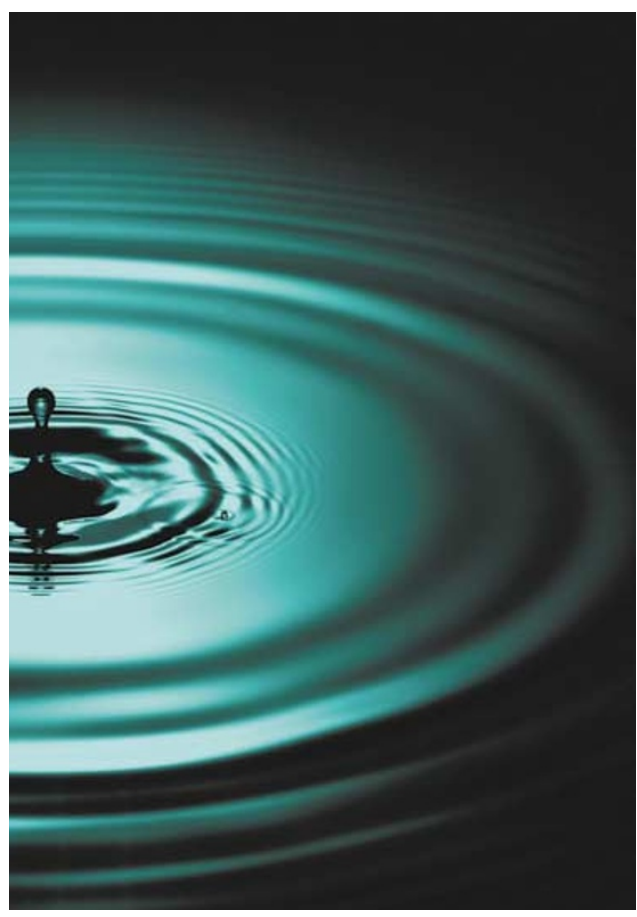

could be expected to lessen the severity of the observed defects. And this is just what the authors saw. For example, macrophages were increased threefold and T cells 3-20-fold in $\operatorname{Trp} 53^{--} \mathrm{Rad} 50^{\mathrm{S} / \mathrm{s}}$ mice compared with Rad50 $5 / s$ mice. Moreover, Trp53--Rad50 $0^{S / s}$ mice developed tumours and died much earlier than $\operatorname{Tr} p 53^{-/-}$ mice alone, again supporting the idea that the Rad50 ${ }^{K 22 M}$ mutation causes genotoxic stress.
A final confirmation came when the authors analysed various indices of genotoxic stress. Levels of phosphorylated histone $\mathrm{H} 2 \mathrm{AX}$ - which correlate with levels of DNA damage - were the same in unirradiated Rad $50^{S / S}$ cells as in irradiated wildtype cells. And karyotype analyses of Rad50 $0^{S / S}$ thymic lymphoma cells revealed increased chromosome breaks and rearrangements (including telomeric short-arm fusions) compared with wild-type cells. So, as the authors conclude, "the data clearly indicate that the Mre11 complex exerts a profound influence on homeostasis in mammalian tissues, even when its checkpoint and DNA recombination functions are not overtly impaired".

Alison Mitchell Editor, Nature Reviews Molecular Cell Biology

(2) References and links ORIGINAL RESEARCh PAPER Bender, C. F. et al. Cancer predisposition and hematopoietic failure in Rad50s/s mice. Genes Dev. 16, 2237-2251 (2002)

FURTHER READING D'Amours, D. \& Jackson, S. P. The Mre11 complex: at the crossroads of DNA repair and checkpoint signalling. Nature Rev. Mol. Cell Biol. 3, 317-327 (2002)

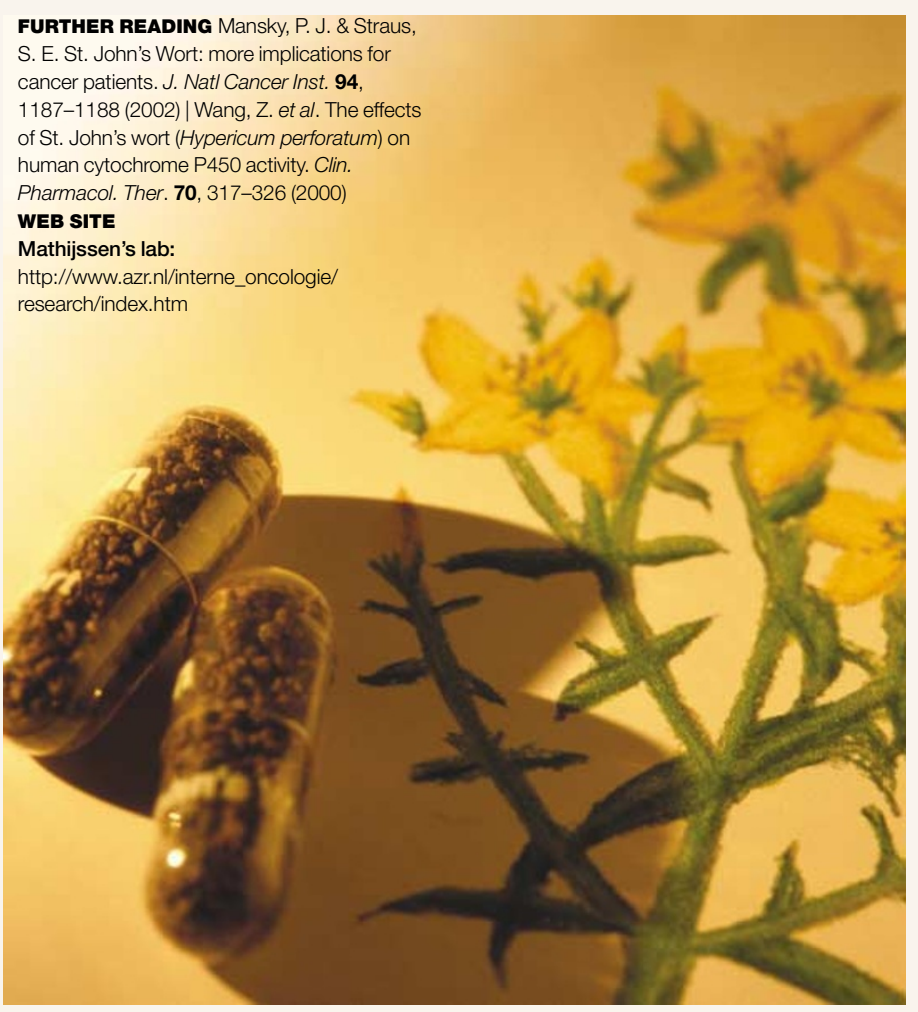

\section{IN BRIEF}

\section{GENETIC SCREENING}

High-throughput retroviral tagging to identify components of specific signalling pathways in cancer.

Mikkers, H. et al. Nature Genet. 32, 153-159 (2002)

This paper, together with two more letters in the September issue of Nature Genetics, describes the first in vivo mammalian genetic screens used to identify genes underlying human cancer. Mikkers et al. show the power of using retroviral insertional mutagenesis, together with the complete mouse genome sequence, by identifying genes that can substitute for Pim1 and Pim2 in lymphomagenesis. This strategy is the mammalian equivalent of the powerful yeast, $D$. melanogaster and C. elegans genetic screens.

\section{ONCOGENES}

\section{RAF/RAS oncogenes and mismatch-repair status.}

Rajagopalan, H. et al. Nature 418, 934 (2002)

Activating mutations in a member of the RAF family, BRAF, have been found in a high proportion of melanomas and in other cancers. Rajagopalan et al. report that BRAF mutations in colorectal cancers occur only in tumours that do not carry mutations in KRAS, and that mutations in BRAF are linked to the ability of these tumours to repair mismatch bases in DNA. These results indicate that mutations in BRAF and KRAS exert equivalent effects in tumorigenesis.

\section{EPIDEMIOLOGY}

On the use of familial aggregation in population-based case probands for calculating penetrance.

Begg, C. B. J. Natl Cancer Inst. 94, 1221-1226 (2002)

High-risk families with multiple cases of breast cancer or case probands are often used in population-based penetrance studies of BRCA1 and BRCA2 mutations. Begg reviews eight published studies and shows that penetrance estimates from case proband studies are biased towards increased risk. This paper highlights the need to improve the methods that are used to predict cancer risk.

\section{ONCOGENES}

Distinct requirements for Ras oncogenesis in human versus mouse cells.

Hamad, N. M. et al. Genes Dev. 16, 2045-2057 (2002)

Oncogenic Ras stimulates three main classes of effector proteins - Rafs, PI3-kinase and RalGEFs - with Rafs generally being the most potent at transforming mouse cells. However, using oncogenic Ras mutants, Hamad et al. found that RalGEF is sufficient for Ras transformation in human cells. The findings indicate that signal-transduction biochemistry studies in mice might have to be revisited to assess applicability to humans, and that RalGEF could be a promising target for anticancer therapies. 